\title{
New calculation schemes for proton-deuteron scattering including the Coulomb interaction
}

\author{
A. Deltuva, ${ }^{1, *}$ A. C. Fonseca, ${ }^{1}$ and P. U. Sauer ${ }^{2}$ \\ ${ }^{1}$ Centro de Física Nuclear da Universidade de Lisboa, P-1649-003 Lisboa, Portugal \\ ${ }^{2}$ Institut für Theoretische Physik, Universität Hannover, D-30167 Hannover, Germany
}

(Received 9 February 2006; published 5 May 2006)

\begin{abstract}
The Coulomb interaction between the protons is included in the description of proton-deuteron scattering using the screening and renormalization approach in the framework of momentum-space integral equations. Two new calculational schemes are presented that confirm the reliability of the perturbative approach for treating the screened Coulomb interaction in high partial waves, used by us in earlier works.
\end{abstract}

DOI: 10.1103/PhysRevC.73.057001

PACS number(s): 21.30.-x, 21.45.+v, 24.70.+s, 25.10.+s

In Refs. [1,2] we included the Coulomb interaction between the protons in the description of proton-deuteron $(p d)$ scattering and of three-nucleon electromagnetic (e.m.) reactions involving ${ }^{3} \mathrm{He}$. The description is based on the Alt-Grassberger-Sandhas (AGS) equations [3] in momentum space. The Coulomb potential is screened, standard scattering theory is applicable, and the resulting scattering amplitudes are corrected by the renormalization technique of Refs. [4,5] to recover the unscreened limit. The special choice of the screened Coulomb potential $w_{R}=w e^{-(r / R)^{n}}, n=4$ being optimal, approximates well the true Coulomb one $w$ for distances $r$ smaller than the screening radius $R$ and simultaneously vanishes rapidly for $r>R$; rather modest values of $R$ are sufficient in order to obtain results that are well converged. However, $R$ is considerably larger than the range of the strong interaction. As a consequence, the calculation of the three-particle transition operators for nuclear plus screened Coulomb potentials requires the inclusion of partial waves with angular momentum much higher than required for the hadronic potential alone. In our previous calculations $[1,2]$ the perturbation theory for high two-particle partial waves, developed in Ref. [6], was used and found to be a very efficient and reliable technical tool for treating the screened Coulomb interaction in those high partial waves. The reliability was established by the stability of the results when varying the dividing line between partial waves included exactly and perturbatively. In this Brief Report we present two alternative calculational schemes that solve $p d$ scattering equations without recourse to the perturbative approach of Ref. [6]. This will enable us to demonstrate directly the reliability of the method for the treatment of the screened Coulomb interaction in high partial waves used in Refs. [1,2].

The first calculational scheme, much like the perturbative approach of Refs. [1,2], is based on the isospin formalism for three nucleons and on the symmetrized AGS equations

$U^{(R)}(Z)=P G_{0}^{-1}(Z)+P T^{(R)}(Z) G_{0}(Z) U^{(R)}(Z)$,

$U_{0}^{(R)}(Z)=(1+P) G_{0}^{-1}(Z)+(1+P) T^{(R)}(Z) G_{0}(Z) U^{(R)}(Z)$

\footnotetext{
*Electronic address: deltuva@cii.fc.ul.pt
}

with $G_{0}(Z)$ being the free resolvent, $P$ the sum of the two cyclic permutation operators, $T^{(R)}(Z)$ the two-particle transition matrix derived from nuclear plus screened Coulomb potentials, and $U^{(R)}(Z)$ and $U_{0}^{(R)}(Z)$ the three-particle transition matrices for elastic and breakup scattering; their dependence on the screening radius $R$ is notationally indicated. The AGS equations are solved as they stand without any perturbative feature. In the practical realization of the solution, i.e., in calculating the Neumann series for the on-shell matrix elements of the operators $U^{(R)}(Z)$ and $T^{(R)}(Z) G_{0}(Z) U^{(R)}(Z)$ and in summing by the Padé method, the most time consuming part is the action of the permutation operator $P$. However, in two-particle partial waves with sufficiently high total angular momentum $I>I_{N}$ the hadronic interaction can safely be neglected and the two-particle transition matrix $T^{(R)}(Z)$ becomes the two-particle screened Coulomb transition matrix $T_{R}(Z)$, which has a nonvanishing contribution only from the proton-proton $(p p)$ interaction. Using the isospin factors of $T_{R}(Z)$ and of $P$ and including the three-nucleon total isospin $\mathcal{T}=\frac{1}{2}$ and $\mathcal{T}=\frac{3}{2}$ states, it can be shown that

$$
T_{R}(Z) G_{0}(Z) P T_{R}(Z)=0
$$

and that general combination of operators is the basic computational building block when calculating the Neumann series for $U^{(R)}(Z)$ and $T^{(R)}(Z) G_{0}(Z) U^{(R)}(Z)$. Thus, the contribution of the $P$ matrix elements between states with $I>I_{N}$ is zero and does not have to be calculated numerically. Since typically $I_{N}$ is 4 to 6 and for the screened Coulomb interaction states up to $I=12$ or even 14 have to be included, this yields a huge saving of computer resources. Compared to our previous calculations based on perturbation theory, the required computing time increases only by a factor of 2 to 4; the corresponding calculations can still be done on a PC, much like the ones of Refs. [1,2].

The second calculational scheme considers protons and neutrons as nonidentical particles. The identity of the two protons is taken into account by using properly antisymmetrized initial and final channel states. The transition operators for elastic/rearrangement scattering and breakup are obtained 


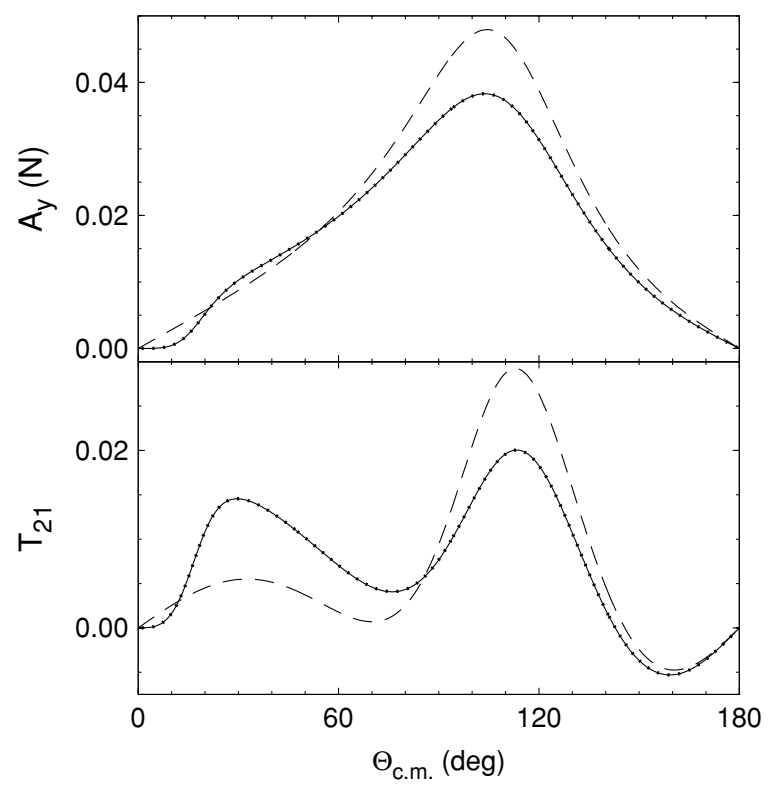

FIG. 1. Nucleon analyzing power $A_{y}(N)$ and deuteron analyzing power $T_{21}$ for $p d$ elastic scattering at $3 \mathrm{MeV}$ proton lab energy as functions of the c.m. scattering angle. The results of the two new calculational schemes without perturbation theory are graphically indistinguishable and are therefore represented by one curve only, the thin solid one; they are compared with the results obtained using perturbative approach of Refs. [1,2] (thick dotted curves). Results without Coulomb (dashed curves) are given as reference for the size of the Coulomb effect.

from standard nonsymmetrized AGS equations

$$
\begin{aligned}
& U_{\beta \alpha}^{(R)}(Z)=\bar{\delta}_{\beta \alpha} G_{0}^{-1}(Z)+\sum_{\sigma} \bar{\delta}_{\beta \sigma} T_{\sigma}^{(R)}(Z) G_{0}(Z) U_{\sigma \alpha}^{(R)}(Z), \\
& U_{0 \alpha}^{(R)}(Z)=G_{0}^{-1}(Z)+\sum_{\sigma} T_{\sigma}^{(R)}(Z) G_{0}(Z) U_{\sigma \alpha}^{(R)}(Z),
\end{aligned}
$$

with $\bar{\delta}_{\beta \alpha}=1-\delta_{\beta \alpha}$. The AGS equations are solved as they stand without any perturbative feature. Here the most time consuming part of calculations is the transformation from the basis where particle $\sigma$ is a spectator to the basis where particle $\beta$ is the spectator, which is closely related to the permutation operator $P$ of the first approach. However, out of the three two-nucleon transition matrices $T_{\sigma}^{(R)}(Z)$ only the one corresponding to a neutron spectator and a $p p$ pair has high angular momentum $I>I_{N}$ components due to the screened Coulomb interaction. Therefore in the transformation of bases the high partial waves with $I>I_{N}$ will only be coupled to low partial waves with $I \leqslant I_{N}$. The efficiency of this calculational scheme is nearly the same as of the first one.

The first calculational scheme is extended to include the $\Delta$-isobar excitation [7] as a mechanism for mediating an

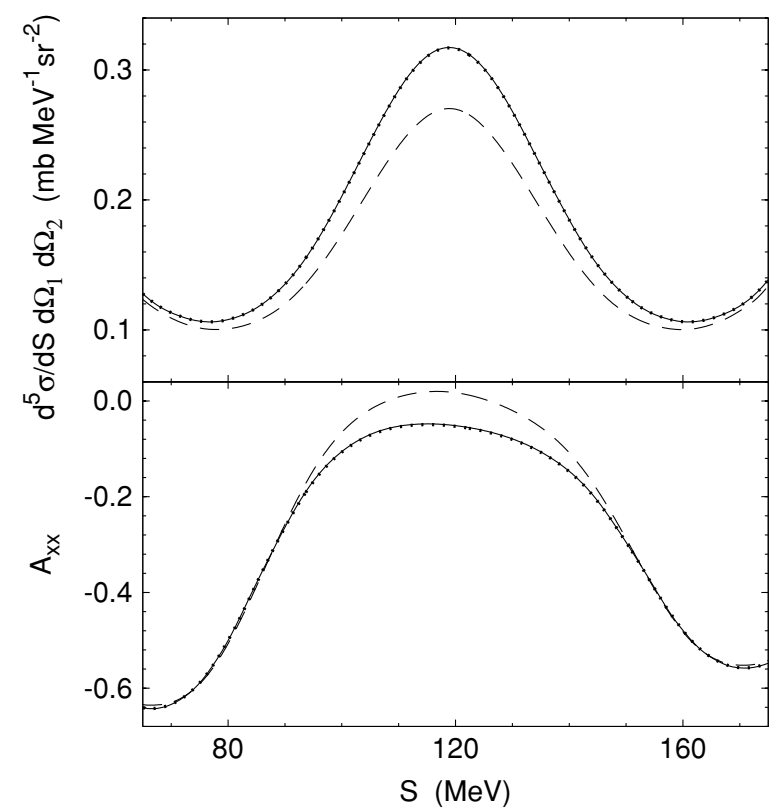

FIG. 2. Differential cross section and deuteron analyzing power $A_{x x}$ for $p d$ breakup at $130 \mathrm{MeV}$ deuteron lab energy in the kinematical configuration $\left(15^{\circ}, 15^{\circ}, 160^{\circ}\right)$ are shown as functions of the arclength $S$ along the kinematical curve. Curves as in Fig. 1.

effective three-nucleon force. In principle, a corresponding extension could be done also for the second scheme. However, we refrain from doing so in this report: Here we are only interested in the technical proof that perturbation theory is highly reliable; furthermore, for the observables at the energies considered the $\Delta$-isobar effects are insignificant. We therefore present only results derived from the purely nucleonic charge-dependent (CD) Bonn potential [8]. Using the two new calculational schemes we recalculated results of Refs. [1,2]. In all cases we found excellent agreement, thereby confirming the reliability of the perturbative approach of Refs. [1,2] for the Coulomb interaction in high partial waves. The examples for elastic $p d$ scattering at $3 \mathrm{MeV}$ proton lab energy and $p d$ breakup at $130 \mathrm{MeV}$ deuteron lab energy are shown in Figs. 1 and 2.

In summary, we presented two new calculational schemes for treating the screened Coulomb interaction in high partial waves without recourse to perturbative approach of Ref. [6] used by us in previous works. A perfect agreement between old and new results confirms the reliability of the method used in Refs. [1,2].

A.D. is supported by the FCT grant SFRH/BPD/ $14801 / 2003$, A.C.F. in part by the FCT grant POCTI/ISFL/2/275, and P.U.S. in part by the DFG grant Sa $247 / 25$.
[1] A. Deltuva, A. C. Fonseca, and P. U. Sauer, Phys. Rev. C 71, 054005 (2005).
[2] A. Deltuva, A. C. Fonseca, and P. U. Sauer, Phys. Rev. C 72, 054004 (2005) 
[3] E. O. Alt, P. Grassberger, and W. Sandhas, Nucl. Phys. B2, 167 (1967).

[4] J. R. Taylor, Nuovo Cimento B 23, 313 (1974); M. D. Semon and J. R. Taylor, Nuovo Cimento A 26, 48 (1975).

[5] E. O. Alt, W. Sandhas, and H. Ziegelmann, Phys. Rev. C 17, 1981 (1978); E. O. Alt and W. Sandhas, ibid. 21, 1733 (1980).
[6] A. Deltuva, K. Chmielewski, and P. U. Sauer, Phys. Rev. C 67, 054004 (2003).

[7] A. Deltuva, R. Machleidt, and P. U. Sauer, Phys. Rev. C 68, 024005 (2003).

[8] R. Machleidt, Phys. Rev. C 63, 024001 (2001). 